\title{
Development of 105 SNP markers in endangered turtle species Pelodiscus sinensis using RAD-seq
}

Qiming Xie (ロ2188149989@qq.com )

School of Animal Science, Anhui Agricultural University, Hefei, China

\section{Fan Liu}

Anhui Agricultural University

Jilong Zhang

Anhui Agricultural University

Xilei Li

Anhui Agricultural University

Tiantian Chen

Anhui Agricultural University

\section{Guoxia Fang}

Feixi County Animal Disease Prevention and Control Center

\section{Rensheng Ma}

Feixi County Animal Disease Prevention and Control Center

\section{Shi-Ping Su}

Anhui Agricultural University

\section{Research Article}

Keywords: Pelodiscus sinensis, RAD-seq, SNP, Conservation genetics

Posted Date: February 11th, 2021

DOI: https://doi.org/10.21203/rs.3.rs-213566/v1

License: (c) (1) This work is licensed under a Creative Commons Attribution 4.0 International License.

Read Full License 


\section{Abstract}

In this study, Restriction site-associated DNA sequencing (RAD-seq) was used to develop singlenucleotide polymorphism (SNP) markers for Pelodiscus sinensis, which is a commercially important species in China. We collected 15 individuals from Anhui Province and RAD-seq data of 20 individuals from NCBI: PRJNA530350 to screen high quality SNP maker through genome-wide. A total of 105 highquality SNP markers were identified. The population genetic statistics showed that the minor allele frequency varied from 0.0571 to 0.3382 , and the observed heterozygosity and expected heterozygosity ranged from 0.1143 to 0.6765 and 0.1078 to 0.4477 , respectively. These novel SNP markers will serve as a valuable genetic tool for future conservation and population genetics studies in $P$. sinensis.

\section{Main Text}

The Chinese soft-shelled turtle (Pelodiscus sinensis) is widely distributed in China, Taiwan, Korea, and North Vietnam and has been used as food and medicine for thousands of years in China (Haitao et al. 2008). Pelodiscus sinensis is regarded as one of the commercially most important aquatic reptile species worldwide and is also an important model organism for evolutionary and developmental biology owing to its soft shell (Cordeiro et al. 2020; Wang et al. 2013). However, in the past decades, the number of $P$. sinensis has markedly reduced owing to human overexploitation and habitat degeneration, and the species is now listed on the IUCN Red List. At present, in China, there are massive $P$. sinensis farms that meet the market demand; however, the animals exhibit several genetic issues such as inbreeding, fixation of deleterious alleles, and loss of genetic diversity. Development of reliable molecular markers is essential for understanding $P$. sinensis population diversity and genetic structure, which are the basis of farm management and genetic resource conservation.

Previous genetic studies of $P$. sinensis have focused on using a few mitochondrial DNA (mtDNA) (Xiong et al. 2019; Xiong et al. 2010) and microsatellite markers (Bu et al. 2014; Zhu et al. 2012). Compared with mtDNA and microsatellite markers, single nucleotide polymorphisms (SNPs) are more repeatable across laboratories, widespread in the genome, flexible for analyzing both neutral and adaptive genetic variation, and easy to acquire and apply using high-throughput sequencing technologies (Kruck et al. 2013). Restriction site-associated DNA sequencing (RAD-seq) can identify large polymorphic genetic markers across the genome in a single, straightforward, and cost-effective experiment (Davey et al. 2011). Presently, the knowledge about genome-wide germplasm resources of soft-shelled turtle remains limited, and genome-wide SNP markers are lacking.

In this study, we used 15 P. sinensis individuals collected from Anhui Province and used the RAD-seq data of 20 individuals from NCBI (PRJNA530350) (Liang et al. 2019). Muscle tissue samples from the 15 individuals were obtained and stored at $-80^{\circ} \mathrm{C}$. RAD library preparation and sequencing were performed by BGI Tech Solutions CO., Ltd. (1010 Room, Building No.7, BGI Parl, No.21, Hongan $3^{\text {rd }}$ Street, Yantian District, Shenzhen, Guangdong Province, China, 518000). The raw read filtration criteria were as follows: remove (a) reads without barcodes, (b) reads without the enzyme cut site, (c) reads with the adapter, (d) 
reads with $\geq 40 \%$ low-quality bases (quality value $\leq 15$ ), and (e) reads with $\geq 10 \%$ unidentified bases. SAMtools of the Burrows-Wheeler Aligner (BWA) tool (Li and Durbin 2009) was used to map the filtered reads to the Chinese soft-shelled turtle reference genome sequence (GCF_000230535.1), and bcftools was used to call SNP variants. To improve the quality of SNPs, vcftools (Danecek et al. 2011) was used to filter the raw SNP set with the following criteria: (a) depth of loci $\leq 15$ and $\geq 3$, (b) GQ of loci $\geq 30$, (c) shared by $95 \%$ sample, and (d) minor allele frequency $\geq 0.05$. Other complex variant events such as InDels were ignored. PLINK was used to exclude those SNP sites that failed the Hardy-Weinberg equilibrium (HWE) test with a $P$ value of 0.000001 and calculate observed heterozygosity $(H o)$, expected heterozygosity $(\mathrm{He})$, and minor allele frequency (MAF). bedtools (Quinlan and Hall 2010) was used to extract a $300 \mathrm{bp}$ flanking sequence from the reference genome according to the SNP sites, and batchprimer3 (You et al. 2008) was used to design primers. Full details of the methods in this study can be found in supplementary material 1.

After quality control, a total of 105 high-quality SNP markers were identified from 10952494 raw SNP sites. The population genetic statistics for each SNP were analyzed (Table 1), and primers were designed for future utilization. The observed heterozygosity and expected heterozygosity varied from 0.1143 to 0.6765 and 0.1078 to 0.4477 , respectively. The minor allele frequency ranged from 0.0571 to 0.3382 . The detailed information of the 105 SNP markers can be found in supplementary material 2 . These novel SNP markers could be a valuable tool for investigating the genetic diversity and population structure of $P$. sinensis, which could facilitate the improvement of farm management and germplasm resource conservation.

\section{Declarations}

\section{Funding}

This work was supported by the Hefei independent innovation policy "loan to supplement" project (No. J2019G37).

\section{Conflict of Interest}

The authors declare that they have no conflict of interest.

\section{Ethics Approval}

This study was followed by the ethical standard of China (GB/T 35892-2018).

\section{Consent to participate}

Not applicable.

\section{Consent for publication}

Not applicable. 


\section{Data Availability}

The RAD-seq data are openly available in NCBI at https://www.ncbi.nlm.nih.gov/sra, PRJNA680995.

\section{Code Availability}

Not applicable.

\section{Supplementary Information}

The detailed process of data analysis and genetic information of 105 SNP markers can be found in Supplementary material 1 and 2 .

\section{ESM Competing Interests}

The authors declare that they have no conflict of interest.

\section{Author Contributions}

Qiming Xie and Shiping Su conceived the presented idea. Qiming Xie and Fan Liu carried out the experiment. Qiming Xie developed the theory and performed the computations. Jilong Zhang and Xilei Li verified the analytical methods. Qiming Xie wrote the manuscript with support from Fan Liu, Jilong Zhang, and Xilei Li. Guoxia Fang and Rensheng Ma provided the experimental samples. Shiping Su supervised the findings of this work. All authors discussed the results and contributed to the final manuscript.

\section{Acknowledgments}

We thank Yang Li for providing computer equipment.

\section{References}

Bu X, Liu L, Nie L (2014) Genetic diversity and population differentiation of the Chinese soft-shelled turtle (Pelodiscus sinensis) in three geographical populations. Biochem Syst Ecol 54:279-284 doi:10.1016/j.bse.2014.02.022

Cordeiro IR, Yu R, Tanaka M (2020) Regulation of the limb shape during the development of the Chinese softshell turtles. Evol Dev 22:451-462 doi:10.1111/ede.12352

Danecek P et al. (2011) The variant call format and VCFtools. Bioinformatics 27:2156-2158 doi:10.1093/bioinformatics/btr330

Davey JW, Hohenlohe PA, Etter PD, Boone JQ, Catchen JM, Blaxter ML (2011) Genome-wide genetic marker discovery and genotyping using next-generation sequencing. Nature Reviews Genetics 12:499-510 doi:10.1038/nrg3012 
Haitao S, Parham JF, Zhiyong F, Meiling H, Feng Y (2008) Evidence for the massive scale of turtle farming in China. Oryx 42:147-150 doi:10.1017/S0030605308000562

Kruck NC, Innes DI, Ovenden JR (2013) New SNPs for population genetic analysis reveal possible cryptic speciation of eastern Australian sea mullet (Mugil cephalus) Molecular Ecology Resources 13:715-725 doi:10.1111/1755-0998.12112

Li H, Durbin R (2009) Fast and accurate short read alignment with Burrows-Wheeler transform Bioinformatics 25:1754-1760 doi:10.1093/bioinformatics/btp324

Liang HW, Wang LH, Sha H, Zou GW (2019) Development and Validation of Sex-Specific Markers in Pelodiscus Sinensis Using Restriction Site-Associated DNA Sequencing Genes 10 doi:10.3390/genes10040302

Quinlan AR, Hall IM (2010) BEDTools: a flexible suite of utilities for comparing genomic features Bioinformatics 26:841-842 doi:10.1093/bioinformatics/btq033

Wang Z et al. (2013) The draft genomes of soft-shell turtle and green sea turtle yield insights into the development and evolution of the turtle-specific body plan Nature Genetics 45:701-+ doi:10.1038/ng.2615

Xiong G, Wang X-Q, Zhou X-W, Zeng D, Chen Z-N, Wang P, Kang L (2019) Genetic variation in the Chinese soft-shell turtles (Pelodiscus spp.) revealed by sequences of mitochondrial Cytb gene Mitochondrial DNA Part A 30:874-879 doi:10.1080/24701394.2019.1693551

Xiong L, Nie L, Li X, Liu X (2010) Comparison research and phylogenetic implications of mitochondrial control regions in four soft-shelled turtles of Trionychia (Reptilia, Testudinata) Genes \& Genomics 32:291298 doi:10.1007/s13258-010-0015-8

You FM et al. (2008) BatchPrimer3: a high throughput web application for PCR and sequencing primer design BMC Bioinformatics 9:253 doi:10.1186/1471-2105-9-253

Zhu L, Li Z, Li J, Feng X, Xie N (2012) Genetic Diversity of Farmed Chinese Soft-Shelled Turtle (Pelodiscus sinensis) Evaluated from Microsatellite Analysis Journal of Animal and Veterinary Advances 11:12171222

\section{Tables}

Table 1 Summary statistics of 105 single nucleotide polymorphism (SNP) markers of Pelodiscus sinensis 


\begin{tabular}{|c|c|c|c|c|c|}
\hline Position & REF & ALT & $M A F$ & Ho & $\mathrm{He}$ \\
\hline NW_005852246.1:55 & A & G & 0.2794 & 0.5588 & 0.4027 \\
\hline NW_005866747.1:181 & $\mathrm{T}$ & C & 0.1176 & 0.2353 & 0.2076 \\
\hline NW_005857380.1:3906 & G & A & 0.3382 & 0.6765 & 0.4477 \\
\hline NW_005854040.1:9429 & A & $\mathrm{T}$ & 0.0857 & 0.1714 & 0.1567 \\
\hline NW_005858158.1:32026 & $\mathrm{T}$ & C & 0.1029 & 0.2059 & 0.1847 \\
\hline NW_005853731.1:38813 & C & $\mathrm{T}$ & 0.0735 & 0.1471 & 0.1362 \\
\hline NW_005851636.1:42529 & $\mathrm{T}$ & C & 0.0882 & 0.1765 & 0.1609 \\
\hline NW_005854351.1:55917 & A & C & 0.0882 & 0.1765 & 0.1609 \\
\hline NW_005854898.1:73656 & C & $\mathrm{T}$ & 0.0571 & 0.1143 & 0.1078 \\
\hline NW_005855443.1:82027 & A & C & 0.0571 & 0.1143 & 0.1078 \\
\hline NW_005853897.1:98550 & C & $\mathrm{T}$ & 0.0882 & 0.1765 & 0.1609 \\
\hline NW_005851906.1:111998 & G & A & 0.0588 & 0.1176 & 0.1107 \\
\hline NW_005856908.1:129462 & C & $\mathrm{T}$ & 0.0735 & 0.1471 & 0.1362 \\
\hline NW_005856362.1:129953 & A & G & 0.1176 & 0.2353 & 0.2076 \\
\hline NW_005856316.1:164154 & G & A & 0.1471 & 0.2941 & 0.2509 \\
\hline NW_005858876.1:203033 & G & A & 0.0588 & 0.1176 & 0.1107 \\
\hline NW_005856075.1:210584 & C & $\mathrm{T}$ & 0.1176 & 0.2353 & 0.2076 \\
\hline NW_005858997.1:239774 & A & G & 0.0735 & 0.1471 & 0.1362 \\
\hline NW_005857910.1:242076 & A & C & 0.0588 & 0.1176 & 0.1107 \\
\hline NW_005854296.1:252072 & C & $\mathrm{T}$ & 0.1029 & 0.2059 & 0.1847 \\
\hline NW_005857744.1:297199 & G & C & 0.0735 & 0.1471 & 0.1362 \\
\hline NW_005856504.1:319845 & G & A & 0.1618 & 0.3235 & 0.2712 \\
\hline NW_005857680.1:323433 & $\mathrm{T}$ & G & 0.1176 & 0.2353 & 0.2076 \\
\hline NW_005855650.1:384770 & G & A & 0.0857 & 0.1714 & 0.1567 \\
\hline NW_005857694.1:407992 & C & A & 0.1029 & 0.2059 & 0.1847 \\
\hline NW_005854923.1:416335 & $\mathrm{T}$ & G & 0.0588 & 0.1176 & 0.1107 \\
\hline NW_005854923.1:416341 & C & G & 0.0588 & 0.1176 & 0.1107 \\
\hline NW_005857006.1:421367 & A & G & 0.1765 & 0.3529 & 0.2907 \\
\hline
\end{tabular}




\begin{tabular}{|llllll|}
\hline NW_005858887.1:475260 & A & G & 0.0588 & 0.1176 & 0.1107 \\
\hline NW_005854747.1:476067 & C & T & 0.0735 & 0.1471 & 0.1362 \\
\hline NW_005871043.1:523453 & G & T & 0.1029 & 0.2059 & 0.1847 \\
\hline NW_005871043.1:523454 & A & C & 0.1029 & 0.2059 & 0.1847 \\
\hline NW_005856824.1:546807 & C & T & 0.0588 & 0.1176 & 0.1107 \\
\hline NW_005854417.1:562053 & A & G & 0.0571 & 0.1143 & 0.1078 \\
\hline NW_005856333.1:562648 & T & A & 0.0735 & 0.1471 & 0.1362 \\
\hline NW_005853643.1:608336 & G & T & 0.0588 & 0.1176 & 0.1107 \\
\hline NW_005855688.1:615542 & G & T & 0.1618 & 0.3235 & 0.2712 \\
\hline NW_005854399.1:618147 & T & C & 0.2714 & 0.5429 & 0.3955 \\
\hline NW_005854399.1:618222 & A & G & 0.2794 & 0.5588 & 0.4027 \\
\hline NW_005854399.1:618253 & T & G & 0.2794 & 0.5588 & 0.4027 \\
\hline NW_005855929.1:624215 & T & G & 0.0571 & 0.1143 & 0.1078 \\
\hline NW_005855694.1:659040 & T & C & 0.0882 & 0.1765 & 0.1609 \\
\hline NW_005858300.1:719759 & C & T & 0.0882 & 0.1765 & 0.1609 \\
\hline NW_005855698.1:795091 & A & T & 0.0588 & 0.1176 & 0.1107 \\
\hline NW_005857290.1:823432 & A & T & 0.2059 & 0.4118 & 0.3270 \\
\hline NW_005853196.1:884143 & G & T & 0.0882 & 0.1765 & 0.1609 \\
\hline NW_005854405.1:896447 & T & C & 0.0882 & 0.1765 & 0.1609 \\
\hline NW_005853644.1:917097 & G & A & 0.0735 & 0.1471 & 0.1362 \\
\hline NW_005855218.1:1025924 & T & C & 0.0588 & 0.1176 & 0.1107 \\
\hline NW_005851747.1:1115210 & G & T & 0.0588 & 0.1176 & 0.1107 \\
\hline NW_005854428.1:1223918 & T & C & 0.2647 & 0.5294 & 0.3893 \\
\hline NW_005853167.1:1227139 & A & T & 0.1618 & 0.3235 & 0.2712 \\
\hline NW_005855792.1:1262551 & G & A & 0.0588 & 0.1176 & 0.1107 \\
\hline NW_005856802.1:1268217 & A & G & 0.0588 & 0.1176 & 0.1107 \\
\hline NW_005871004.1:1270305 & T & C & 0.1912 & 0.3824 & 0.3093 \\
\hline NW_005852159.1:1315537 & A & G & 0.1618 & 0.3235 & 0.2712 \\
\hline NW005856823.1:1330905 & C & A & 0.0588 & 0.1176 & 0.1107 \\
\hline NW & & & & \\
\hline NW & & & & \\
\hline NW & & & \\
\hline
\end{tabular}




\begin{tabular}{|llllll|} 
NW_005854781.1:1427409 & T & A & 0.1471 & 0.2941 & 0.2509 \\
\hline NW_005855476.1:1508482 & G & A & 0.1176 & 0.2353 & 0.2076 \\
\hline NW_005858753.1:1620758 & G & A & 0.0882 & 0.1765 & 0.1609 \\
\hline NW_005859202.1:1628000 & G & A & 0.1029 & 0.2059 & 0.1847 \\
\hline NW_005871004.1:1630732 & T & G & 0.0588 & 0.1176 & 0.1107 \\
\hline NW_005855236.1:1678320 & C & T & 0.0735 & 0.1471 & 0.1362 \\
\hline NW_005853122.1:1680360 & C & G & 0.0735 & 0.1471 & 0.1362 \\
\hline NW_005852052.1:1813181 & G & A & 0.2059 & 0.4118 & 0.3270 \\
\hline NW_005852052.1:1813185 & C & T & 0.2059 & 0.4118 & 0.3270 \\
\hline NW_005857440.1:1876366 & G & A & 0.1912 & 0.3824 & 0.3093 \\
\hline NW_005853738.1:1881846 & C & T & 0.1286 & 0.2571 & 0.2241 \\
\hline NW_005855397.1:1897760 & T & C & 0.0588 & 0.1176 & 0.1107 \\
\hline NW_005855397.1:1897783 & T & C & 0.0735 & 0.1471 & 0.1362 \\
\hline NW_005851563.1:2179842 & T & C & 0.1029 & 0.2059 & 0.1847 \\
\hline NW_005856064.1:2201529 & G & A & 0.0735 & 0.1471 & 0.1362 \\
\hline NW_005856064.1:2201558 & C & T & 0.0735 & 0.1471 & 0.1362 \\
\hline NW_005856064.1:2201567 & A & G & 0.0735 & 0.1471 & 0.1362 \\
\hline NW_005858084.1:2204830 & C & T & 0.0571 & 0.1143 & 0.1078 \\
\hline NW_005857732.1:2240113 & A & C & 0.0571 & 0.1143 & 0.1078 \\
\hline NW_005857401.1:2240213 & G & A & 0.0735 & 0.1471 & 0.1362 \\
\hline NW_005852467.1:2246898 & G & A & 0.0882 & 0.1765 & 0.1609 \\
\hline NW_005852467.1:2246902 & T & C & 0.0882 & 0.1765 & 0.1609 \\
\hline NW_005853176.1:2506285 & G & A & 0.1324 & 0.2647 & 0.2297 \\
\hline NW_005852769.1:2548930 & T & A & 0.1324 & 0.2647 & 0.2297 \\
\hline NW_005859159.1:2600139 & A & T & 0.0588 & 0.1176 & 0.1107 \\
\hline NW_005854634.1:2883497 & A & G & 0.1618 & 0.3235 & 0.2712 \\
\hline NW_005853523.1:2999985 & C & T & 0.0588 & 0.1176 & 0.1107 \\
\hline NW_005851929.1:3140999 & C & T & 0.3382 & 0.6765 & 0.4477 \\
\hline NW555792.1:3166874 & C & G & 0.0588 & 0.1176 & 0.1107 \\
\hline NW & & & & \\
\hline NW & & & & \\
\hline NW & & & \\
\hline
\end{tabular}




\begin{tabular}{|llllll|} 
NW_005854896.1:3706567 & C & T & 0.0882 & 0.1765 & 0.1609 \\
\hline NW_005851925.1:3737622 & C & A & 0.0588 & 0.1176 & 0.1107 \\
\hline NW_005853657.1:3836504 & A & T & 0.0588 & 0.1176 & 0.1107 \\
\hline NW_005855489.1:4002735 & A & G & 0.0882 & 0.1765 & 0.1609 \\
\hline NW_005855637.1:4174003 & T & C & 0.1176 & 0.2353 & 0.2076 \\
\hline NW_005871043.1:4270476 & G & C & 0.0882 & 0.1765 & 0.1609 \\
\hline NW_005871043.1:4270505 & C & T & 0.0882 & 0.1765 & 0.1609 \\
\hline NW_005858987.1:4749868 & G & T & 0.0571 & 0.1143 & 0.1078 \\
\hline NW_005858573.1:5726004 & T & C & 0.0588 & 0.1176 & 0.1107 \\
\hline NW_005858573.1:6235628 & G & A & 0.1176 & 0.2353 & 0.2076 \\
\hline NW_005858573.1:6235633 & A & G & 0.1176 & 0.2353 & 0.2076 \\
\hline NW_005855477.1:7581107 & A & G & 0.1324 & 0.2647 & 0.2297 \\
\hline NW_005853697.1:8051021 & C & T & 0.0588 & 0.1176 & 0.1107 \\
\hline NW_005871009.1:8713326 & C & T & 0.0588 & 0.1176 & 0.1107 \\
\hline NW_005853106.1:8919331 & C & T & 0.0588 & 0.1176 & 0.1107 \\
\hline NW_005854622.1:9141592 & G & A & 0.0714 & 0.1429 & 0.1327 \\
\hline NW_005854622.1:9141593 & C & T & 0.0571 & 0.1143 & 0.1078 \\
\hline NW_005871025.1:9733061 & T & A & 0.0588 & 0.1176 & 0.1107 \\
\hline NW_005855477.1:11005396 & G & A & 0.0882 & 0.1765 & 0.1609 \\
\hline
\end{tabular}

Note: REF, major allele; ALT, minor allele; MAF, minor allele frequency; $\mathrm{Ho}$, observed heterozygosity; $\mathrm{He}$, expected heterozygosity.

\section{Supplementary Files}

This is a list of supplementary files associated with this preprint. Click to download.

- Supplymentarymaterials1.docx

- Supplymentarymaterials2.xlsx 